\title{
Management von Tumoren mit neuroendokriner Differenzierung
}

\author{
Alexander Haug ${ }^{\mathrm{a}}$ Thomas Pfluger $^{\mathrm{b}}$ Philipp A. Schnabel ${ }^{\mathrm{c}} \quad$ Okan Zaba $^{\mathrm{d}}$ \\ ${ }^{a}$ Klinik und Poliklinik für Nuklearmedizin, Ludwig-Maximilians-Universität München, \\ ${ }^{b}$ Klinik und Poliklinik für Nuklearmedizin, Klinikum der Universität München - Campus Innenstadt, \\ ${ }^{c}$ Pathologisches Institut, Sektion Thoraxpathologie, Universitätsklinikum Heidelberg, \\ ${ }^{\mathrm{d}}$ Pneumologische Klinik, Evangelische Lungenklinik Berlin, Deutschland
}

\section{Wann ist ein Tumor neuroendokrin - und wann ist diese Differenzierung therapierelevant?}

Grundvoraussetzung für die Identifizierung eines neuroendokrinen Tumors (NET) ist der histopathologische Nachweis neuroendokriner Charakteristika. Zusätzliche Anhaltspunkte geben immunhistochemisch charakterisierte neuroendokrine Marker sowie elektronenmikroskopisch verifizierte neuroendokrine Granula. Therapierelevant ist die Differenzierung, ob ein NET vorliegt bei den Karzinoiden der Lunge, dem kleinzelligen Lungenkarzinom (SCLC) sowie den großzelligen neuroendokrinen Karzinomen (LCNEC).

\section{Neuroendokrine Differenzierung}

Ein typisches histomorphologisches Charakteristikum neuroendokriner Lungentumore ist die trabekuläre Bauweise. Darüber hinaus finden sich häufig eine randständige Palisadenstellung von Zellen bzw. Zellkernen, (Pseudo-)Rosetten und ein organoides «Nesting». Der Nachweis einer neuroendokrinen Differenzierung ist am bioptischen Material, das einen wesentlich kleineren Ausschnitt des Tumors repräsentiert, meist deutlich schwieriger als am Operationspräparat. Ergänzend können hier immunhistochemisch bestimmte neuroendokrine Marker herangezogen werden, wie z.B. CD56, Chromogranin A oder Synaptophysin. Vergleichsweise wenig spezifisch ist der Nachweis der neuronenspezifischen Enolase (NSE) als Tumormarker. Chromogranin A ist üblicherweise in gut differenzierten NET wie z.B. Karzinoiden stärker exprimiert als in SCLC und LCNEC. Beim LCNEC soll nach der Klassifikation der World Health Organization (WHO) [1] zusätzlich zur neuroendokrinen Histomorphologie mindestens ein neuroendokriner Marker außer NSE immunhistochemisch deutlich positiv sein. Darüber hinaus müssen über 11 Mitosen pro 10 hochauflösende Gesichtsfelder (>11/10 HPF) vorliegen [1].
Typische und atypische Karzinoide

Karzinoide sind mit einer vergleichsweise guten Prognose assoziiert. Die Patienten werden zu einem hohen Prozentsatz durch eine Operation geheilt. Das Metastasierungspotenzial ist gering. Die 5-Jahres-Überlebensrate für Patienten mit typischem Karzinoid liegt bei $87-100 \%$ und beim Nachweis eines atypischen Karzinoids bei $61-88 \%$. Gleichwohl lässt sich im Einzelfall die definitive Prognose nicht immer zuverlässig voraussagen. Warum einzelne Karzinoid-Patienten eine deutlich schlechtere Prognose haben als das Gros der Patienten, ist unklar und Gegenstand weiterer Untersuchungen. Erfolgversprechend scheint hier ein kombiniertes Grading-System, das die Mitoserate und den Proliferationsindex (PI) berücksichtigt: Das System wurde zunächst für Patienten mit gastrointestinalen NET validiert [2] und später auf die pulmonalen Karzinoide adaptiert [3]. Tatsächlich zeigte sich eine höhere prognostische Sensitivität für Patienten mit pulmonalem Karzinoid und damit ein besseres Abbild der klinischen Wirklichkeit $[3,4]$.

\section{Kleinzelliges Lungenkarzinom}

Patienten mit SCLC haben in der Regel eine sehr schlechte Prognose. Nur wenige Patienten erreichen ein Langzeitüberleben. Dennoch gibt es Ausnahmen. Neben Resistenzmechanismen werden Tumor-Matrix-Interaktionen diskutiert, die für den unterschiedlichen Verlauf einzelner SCLC verantwortlich sind. Differente pathogenetische Variablen sind möglicherweise eine Erklärung für eine unterschiedliche Prognose der Patienten. Eine Untersuchung aus Heidelberg [5] weist darauf hin, dass die Pathogenese des SCLC möglicherweise auch virusbedingt sein kann: So kommt es beim SCLC häufiger zu einer Promoter-Hypermethylierung des TumorSuppressorgens RASSF1A (Ras association domain-containing protein). Die Hypermethylierung kann mit einer Polyo-

\begin{tabular}{ll}
\hline KARGER & (1) 2013 S. Karger GmbH, Freiburg \\
$0378-584 X / 13 / 3618-0009 \$ 38.00 / 0$ \\
Fax +49 761 4520714 \\
$\begin{array}{l}\text { Information@Karger.com } \\
\text { www.karger.com }\end{array}$ & $\begin{array}{l}\text { Accessible online at: } \\
\text { www.karger.com/onk }\end{array}$
\end{tabular}


mavirus-Infektion assoziiert sein. In der Heidelberger Untersuchung wurden bei einigen SCLC-Patienten sowohl eine Hypermethylierung des RASSF1A-Promoters als auch eine Polyomavirus-Infektion nachgewiesen.

\section{Großzelliges neuroendokrines Karzinom}

Patienten mit LCNEC haben eine ähnliche oder noch schlechtere Prognose als jene mit SCLC. Das Problem ist darüber hinaus die zuverlässige Diagnosestellung, die insbesondere an Biopsien schwierig ist. Im therapeutischen Bereich stellt sich die Frage, ob LCNEC-Patienten genauso behandelt werden sollten wie jene mit SCLC oder ob andere Therapieoptionen geprüft werden sollten.

Die Diagnosestellung erfolgt anhand der bereits beschriebenen Kriterien. Differentialdiagnostisch müssen die LCNEC gegen die nichtkleinzelligen Lungenkarzinome (NSCLC) mit neuroendokriner Differenzierung (NE-NSCLC) abgegrenzt werden [4]. Entscheidend ist, dass die NE-NSCLC histologisch (und immunhistochemisch) die Kriterien eines NSCLC erfüllen und keine dominante neuroendokrine Morphologie aufweisen, sondern nur eine partielle und meist schwächere Expression eines oder mehrerer neuroendokriner Marker.

Ob die partielle Expression neuroendokriner Marker die Prognose der Patienten beeinflusst, ist umstritten. In einer kürzlich erschienenen Arbeit [6] war die partielle neuroendokrine Markerexpression ohne prognostische Relevanz und damit auch therapeutisch nicht relevant. Vor diesem Hintergrund sollte einer partiellen neuroendokrinen Differenzierung bei histologisch gesichertem NSCLC aktuell keine therapeutische Bedeutung beigemessen und der Tumor nicht als neuroendokrin klassifiziert werden [4].

\section{Differentialdiagnostik und Therapiestratifikation mit nuklearmedizinischen Verfahren}

Grundlage der Darstellung von Lungentumoren mit nuklearmedizinischen Verfahren sind der Glukosestoffwechsel, der sich mit dem Glukose-Analogon 18-F-Fluordesoxyglukose (FDG) darstellen lässt, der Nachweis der Decarboxylase-Aktivität mittels F-18-DOPA-PET sowie der Nachweis von Somatostatin-Rezeptoren (SSTR). Bislang sind fünf SSTR-Subtypen bekannt, von denen Subtyp 2 und 5 über 90\% der Fälle ausmachen. Genau diese beiden Subtypen lassen sich mit nuklearmedizinischen Methoden nachweisen. Der Nachweis der Decarboxylase-Aktivität mit F-18-DOPA-PET konkurriert derzeit mit dem Nachweis der SSTR-Expression.

Klassischerweise wurde die SSTR-Expression mit In-111Octreotid nachgewiesen. Eine bessere Bildauflösung wird jedoch mit dem Technetium(Tc)-Tracer und dem Einsatz von Tc-99m EDDA/HYNIC-TATE (Tc-99m EHT) erreicht. Neu ist Gallium(Ga)-68-DOTA-TATE, das in klinischen Studien bei gut differenziertem NET und einem PI von bis zu $20 \%$ eine sehr gute Bildauflösung in der Positronenemissionstomo- graphie (PET) erreicht. Eine sehr hohe Nachweisrate an SSTR zeigt sich unter anderem bei den (pulmonalen) Karzinoiden und den funktionell nicht aktiven NET.

Die drei Tracer - In-111-Octreotid, Tc99m EHT, Ga-68DOTA-TATE - unterscheiden sich im radionuklidtragenden Teil, weniger im Somatostatinanalogon. Die Rezeptorbindung der drei Tracer ist daher als identisch anzusehen. Unterschiede zeigen sich bei der Bildauflösung. Ist als sensitivste Methode keine PET-Bildgebung möglich, kann der Tc-Tracer verwendet werden.

Mit Ga-68-DOTA-TATE bzw. dem DOTA-TATE-PET lassen sich bei gut differenzierten NET mit einem PI von maximal 20\% selbst sehr kleine Knochen- und Knochenmarksmetastasen zuverlässig nachweisen, die in der Computertomographie (CT) nicht darstellbar sind. Als minimale Nachweisgrenze von Knochen- bzw. Knochenmarksmetastasen gelten in der Nuklearmedizin Läsionen von $1 \mathrm{~cm}$; diese Grenze lässt sich mit DOTA-TATE-PET nach unten verschieben. Vorteile hat das DOTA-TATE-PET daher bei Patienten mit gut differenziertem NET und multiplen Metastasenherden. Hier lässt sich zusätzlich zu den primären Tumormanifestationen in der Lunge auch die Ausdehnung der Metastasenlast zuverlässig darstellen. Da die PET-Untersuchung jedoch keine ausreichende Sensitivität zum Nachweis kleiner Lungenrundherde erreicht, bietet sich der Einsatz einer PETCT an, um die extrapulmonalen Metastasen mittels PET und die pulmonalen Metastasen mittels CT abzubilden.

Die hohe Bildauflösung dank DOTA-TATE bestätigte sich in einer Vergleichsuntersuchung bei 25 Patienten mit gut differenziertem NET [7]: Hier wurde die Sensitivität des GaDOTA-TATE PET-CT mit dem F-DOPA PET-CT verglichen. Bei 13 der 25 Patienten erhöhte DOTA-TATE die diagnostische Aussage. 11 F-DOPA-negative Patienten waren DOTA-TATE-positiv und bei weiteren 2 Patienten konnten zusätzliche Metastasenherde identifiziert werden. Die Sensitivität von DOTA-TATE erreichte im Skelett, den Lymphknoten, der Lunge und im Primärtumor 100\% und in der Leber 96\%. F-DOPA erreichte nur im Primärtumor 100\% Sensitivität; in den anderen genannten Organsystemen lag die Sensitivität bei $48-60 \%$.

Bei den schlecht differenzierten bzw. entdifferenzierten NET wie z.B. dem SCLC bringt die DOTA-TATE-PET dagegen keinen Vorteil. Hier ist die FDG-PET-Untersuchung das Diagnostikum der ersten Wahl.

\section{Differentialtherapie mit Radiopeptiden}

Die Behandlung mit Radiopeptiden ist bei den pulmonalen NET für Patienten mit typischem und atypischem Karzinoid eine Option. Bei allen anderen pulmonalen NET hat die nuklearmedizinische Therapie keine Bedeutung. Die Datenlage ist bislang sehr limitiert. Die meisten Daten liegen zu den gastroenteropankreatischen NET (GEP-NET) vor. Während Pa- 
tienten mit Karzinoid im frühen Stadium eine sehr gute Prognose haben, ist die Prognose der fernmetastasierten Patienten mit einer medianen Überlebenszeit mit etwa 16 Monaten extrem schlecht. Die nuklearmedizinische Therapie mit den beiden Somatostatin-Analoga DOTA-TATE und DOTA-TOC bietet diesen Patienten eine neue Therapieoption.

\section{DOTA-TATE versus DOTA-TOC}

Beide Somatostatin-Analoga binden spezifisch an den SSTR-2. Während es für die Diagnostik keine Rolle spielt, welches Somatostatin-Analogon eingesetzt wird, weil Sensitivität und Speicherintensität beider Substanzen vergleichbar sind, zeigen sich für die Therapie Unterschiede: DOTATATE induziert aufgrund einer höheren Affinität zum SSTR-2 eine um den Faktor 2,1 längere Tumorresistenzzeit bei gleichzeitig nur geringfügig längerer Resistenzzeit der Nieren (Faktor 1,4). Darüber hinaus erreicht DOTA-TATE eine höhere Speicherung im Tumor, was im Tiermodell dazu führte, dass mit DOTA-TATE eine höhere Strahlendosis erreicht wurde (46 Gy vs. 17 Gy) [8-10].

Mit ${ }^{90}$ Yttrium $\left({ }^{90} \mathrm{Y}\right)$ und ${ }^{177}$ Lutetium $\left({ }^{177} \mathrm{Lu}\right)$ stehen zwei unterschiedliche Radionuklide zu Verfügung: ${ }^{90} \mathrm{Y}$ ist ein reiner Betastrahler mit einer Halbwertszeit (HWZ) von 64 Stunden (knapp 3 Tage) und einer sehr hohen Beta-Energie $\left(E_{\max }\right.$ 2,270 keV). Die Reichweite der Elektronen ist daher mit maximal $11 \mathrm{~mm}$ vergleichsweise weit. ${ }^{177} \mathrm{Lu}$ ist dagegen ein kombinierter Beta-/Gammastrahler. Da es sich um einen weichen Betastrahler handelt, ist die Reichweite sehr kurz mit im Mittel $0,3 \mathrm{~mm}$ und maximal 1,6 $\mathrm{mm}$.

\section{Therapiedurchführung}

Voraussetzung für eine Radionuklidtherapie ist eine ausreichende SSTR-Speicherung. Die Tumoren sollten eine über die Leber hinausgehende Speicherintensität aufweisen. Die Patienten müssen sich in einer palliativen Therapiesituation befinden, mit dokumentiertem Tumorprogress. Ausnahmen sind Hochrisikopatienten, z.B. mit Gewichtsverlust und/oder hepatischer Tumorlast, sowie solche mit funktionell aktivem NET, die trotz Biotherapie symptomatisch sind. Da die Radionuklidtherapie mit einer relevanten Nephro- und Hämatotoxizität einhergehen kann, müssen die Patienten eine ausreichende Nierenfunktion (glomeruläre Filtrationsrate (GFR) > $40 \mathrm{ml} /$ min; Kreatinin < 1,8 mg/dl; MAG3-Szintigraphie) und eine ausreichende Hämatopoese (Thrombozyten > 80 G/l; Leukozyten > 2,5 G/l; Hämoglobin > 10 g/dl) aufweisen. Eine eventuell vorbestehende Behandlung mit Somatostatin-Analoga muss mindestens 4 Wochen vor Therapiebeginn abgesetzt werden. Nur im Einzelfall kann - speziell bei GEP-NET-Patienten - eine neoadjuvante Radionuklidtherapie indiziert sein, um die Patienten in eine operable Situation zu überführen.

Die Behandlung erfolgt stationär. Die Patienten erhalten bis zu vier Zyklen mit $7400 \mathrm{MBq}{ }^{177} \mathrm{Lu}$-DOTA-TATE oder $3700 \mathrm{MBq}{ }^{90}$ Y-DOTA-TOC im Abstand von jeweils 6-12 Wochen. Obligat ist eine Co-Infusion von Aminosäurelösungen
(Arginin/Lysin), um zu verhindern, dass die SomatostatinAnaloga in die proximalen Nierentubuli rückresorbiert werden, was die Strahlenbelastung der Niere deutlich erhöhen würde. Durch die Co-Infusion wird die Strahlenbelastung um etwa $40 \%$ reduziert. Nachteil der Co-Infusion sind Übelkeit/ Erbrechen bei etwa $25 \%$ der Patienten sowie ein zum Teil hoher Anstieg der Kaliumspiegel bei einigen Patienten.

\section{Limitierte Studienlage}

Vor 3 Jahren publizierten Bushnell et al. [11] eine prospektive Studie mit 90 Hochrisikopatienten mit gut differenzierten NET des Ileums. Alle Patienten waren hepatisch metastasiert, was bei ilealen NET ein unabhängiger Risikofaktor ist. Alle Patienten waren trotz Biotherapie symptomatisch und bei Studieneinschluss progredient. Nach Behandlung mit drei Zyklen $\left(4,4 \mathrm{GBq}{ }^{90}\right.$ Y-DOTA-TOC) betrug das mediane progressionsfreie Überleben 16,3 Monate bei einer medianen Gesamtüberlebenszeit von über 2 Jahren (26,9 Monate). Gleichzeitig kam es zu einer deutlichen und anhaltenden Reduktion der tumorbedingten Beschwerden und damit einhergehend $\mathrm{zu}$ einer Besserung der Lebensqualität der Patienten.

Bei $70 \%$ der Patienten hatte sich die Erkrankung unter ${ }^{90}$ Y-DOTA-TOC stabilisiert, bei $4 \%$ kam es zur Tumorrückbildung. Vergleichbare Ergebnisse zeigten sich noch in zwei weiteren klinischen Studien [12,13], darunter eine Studie aus der Schweiz mit 1109 Patienten [13]. Hier erreichten sogar $34 \%$ der Patienten eine Reduktion des Tumorvolumens, deren Größenordnung allerdings nicht näher spezifiziert war. Die mediane Gesamtüberlebenszeit der Patienten mit GröBenreduktion betrug 45,6 Monate.

Eine niederländische Arbeitsgruppe erreichte mit ${ }^{177} \mathrm{Lu}-$ DOTA-TATE bei 310 NET-Patienten eine mediane progressionsfreie Zeit von 40 Monaten und eine mediane Gesamtüberlebenszeit von 46 Monaten. Von den 310 Patienten erzielten $30 \%$ eine objektive Tumorremission, darunter auch einzelne komplette Remissionen. Bei 16\% der Patienten kam es zu einer Minor Response und bei 35\% zu einer vorübergehenden Krankheitsstabilisierung. Primär progredient waren $20 \%$. Interessanterweise profitierten die Patienten mit Minor Response und Stabilisierung prognostisch genauso von der Behandlung wie die Patienten mit objektiver Remission und überlebten deutlich länger als die primär progredienten Patienten. Auch in dieser Studie besserte sich die Lebensqualität der Patienten [14, 15].

Nur wenige Studien berichten über den Therapieverlauf bei Patienten mit pulmonalem NET. In der Studie von Bodei et al. [16] waren auch 84 Patienten mit pulmonalem NET mit ${ }^{177} \mathrm{Lu}-$ DOTA-TATE behandelt worden. Bei $29 \%$ kam es zu einem Tumoransprechen. 8 Patienten hatten einen NET des Thymus, von denen 3 Patienten auf die Behandlung ansprachen.

Günstig für ein Therapieansprechen mit mindestens einer Stabilisierung der Erkrankung unter Radionuklidtherapie sind eine geringe oder keine hepatische Tumorlast, ein Karnofsky-Index $>70 \%$, kein Gewichtsverlust vor Therapiebe- 
ginn, keine ossären Filiae, ein funktionell inaktiver Primärtumor und eine hohe Speicherung in Octreoscan bzw. SSTRPET. Unklar ist, ob eine vorherige Chemotherapie prognostisch ungünstig ist. Es verdichten sich die Hinweise, dass dies nicht der Fall ist. Der Ki-67-Wert spielt zwar offiziell für die Klassifizierung der pulmonalen NET keine Rolle, hat aber insofern Bedeutung für die Radionuklidtherapie als sich bis zu einem Ki-67-Wert von 20\% keine Unterschiede im Therapieansprechen zeigen. Liegt der Ki-67-Wert über 20\% und handelt es sich um einen G3-Tumor, wird die Ansprechrate deutlich schlechter.

Die Radionuklidtherapie ist eine vergleichsweise nebenwirkungsarme Therapie. Etwa 10\% der Patienten entwickeln eine relevante Hämatotoxizität - unabhängig davon, welches Radiopharmakon eingesetzt wird. Unterschiede zeigen sich bei der Nephrotoxizität, die unter ${ }^{90} \mathrm{Y}$-DOTA-TOC in etwa $10 \%$ klinische Relevanz erreicht und damit deutlich höher liegt als unter ${ }^{177}$ Lu-DOTA-TATE mit 1-2\% [11-14, 16]. Ein erhöhtes nephrotoxisches Risiko haben Patienten mit nicht eingestelltem Hypertonus oder Diabetes mellitus sowie einem Abfall der Kreatininclearance [14, 17].

Eine neue Entwicklung im Rahmen der Radionuklidtherapie ist die Kombination des Radiopharmakons mit strahlensensibilisierenden Substanzen. Studien mit Capecitabin [18] und Capecitabin/Temozolamid [19] zeigen jeweils erfolgversprechende Ergebnisse. Ein interessanter Ansatz ist die intraarterielle Applikation der Radionuklidtherapie mit dem Ziel, am Metastasierungsort eine höhere Strahlendosis zu erzielen. Auch hier zeigen sich erste vielversprechende Ansprechraten, ohne dass die Toxizität erhöht scheint [20].

\section{Neuroendokrine Lungenkarzinome: Neue Optionen der Systemtherapie}

\section{Kleinzelliges Lungenkarzinom}

Seit den 1990er-Jahren hat es beim SCLC keinen Fortschritt bei der systemischen Behandlung gegeben. Standard sind nach wie vor die seit Jahren eingesetzten Chemotherapieregime mit Platin/Etoposid, Topotecan sowie Kombinationstherapien mit Anthrazyklinen, Alkylantien, Taxanen und Irinotecan. In der klinischen Entwicklung befinden sich der Multikinase-Hemmer Vandetanib, das synthetische Anthrazyklin Amrubicin und der Histon-Deacetylase(HDAC)-Inhibitor Panobinostat.

Vandetanib erreichte bislang in kontrollierten klinischen Studien weder als Erhaltungstherapie noch als «Add-on» zur Induktionstherapie einen medianen Überlebensvorteil für die Patienten. Die mediane progressionsfreie Zeit war unter der Erhaltungstherapie mit Vandetanib tendenziell verlängert [21]. Vielversprechender sind die ersten Studienergebnisse mit Amrubicin. Als Zweitlinientherapie eingesetzt erreichte Amrubicin in einer allerdings kleinen Phase-II-Studie Wirksamkeitsvorteile gegenüber Topotecan bei Patienten mit Pla- tin-sensiblem SCLC [22]. Die Zweitlinientherapie mit Panobinostat erzielte in einer multizentrischen Phase-II-Studie bei $50 \%$ der rezidivierten SCLC-Patienten eine vorübergehende Krankheitsstabilisierung, aber keine objektive Tumorremission [23]. In einer derzeit laufenden kontrollierten klinischen Studie wird Panobinostat als Add-on zur Erstlinientherapie eingesetzt. Ergebnisse liegen noch nicht vor.

\section{Großzelliges neuroendokrines Karzinom}

Bis heute gibt es keine evidenzgestützte internationale Empfehlung zur systemischen Behandlung von Patienten mit LCNEC. Etabliert hat sich im klinischen Alltag die Behandlung mit Cisplatin/Etoposid. In einer aktuellen Analyse aus Frankreich sprachen 35,7\% der Patienten auf die Behandlung mit Cisplatin/Etoposid an und blieben im Median 5,2 Monate ohne Progress bei einer medianen Gesamtüberlebenszeit von 7,7 Monaten, was die extrem ungünstige Prognose der LCNEC-Patienten widerspiegelt [24].

Ein hoffnungsvoller neuer Ansatz ist die Hemmung von mTOR (mammalian target of rapamycin). mTOR ist ein für die Pathogenese zahlreicher Tumorarten wichtiges Target, da der PI3K/AKT/mTOR-Signalweg über mTOR läuft und bei dessen Blockade unterbrochen wird. In einer derzeit laufenden multizentrischen Phase-II-Studie (CRAD001KDE37) bei LCNEC-Patienten im Stadium IV wird der mTOR-Inhibitor RAD001 (Everolimus) zusätzlich zur Erstlinientherapie mit Carboplatin/Paclitaxel eingesetzt. Primäre Studienendpunkte sind die mediane progressionsfreie und die Gesamtüberlebenszeit. Ergebnisse liegen noch nicht vor.

\section{Pulmonale Karzinoide}

Für Patienten mit nicht mehr operablem pulmonalem Karzinoid bieten sich nuklearmedizinische Therapien an, und falls diese nicht infrage kommen oder ausgereizt sind, ist die Chemotherapie eine Option: Mit den derzeit verfügbaren Chemotherapie-Kombinationen (Streptozotocin/5-Fluoruracil, Streptozotocin/Doxorubicin, Doxorubicin/Paclitaxel oder Cisplatin/Etoposid) werden jeweils Ansprechraten von 20$25 \%$ erreicht; die Daten stammen zum Teil von nichtpulmonalen NET.

In der klinischen Prüfung befindet sich auch hier die mTOR-Inhibition. In der offenen multizentrischen einarmigen Phase-II-Studie RAMSETE (NCT00688623) [25] sind bislang 73 der Patienten mit fortgeschrittenem nichtpankreatischen NET (ohne Karzinoid-Syndrom) jeglicher Therapielinie auswertbar, darunter 16 Patienten mit pulmonalem Karzinoid: Eine partielle Remission bzw. vorübergehende Stabilisierung der Erkrankung erreichten 5\% bzw. 65\% aller ausgewerteten Patienten bei einer medianen progressionsfreien Zeit von 280 Tagen. Die Patienten mit pulmonalen Tumoren blieben im Median 195 Tage ohne Progress. Die deutlich kürzere mediane progressionsfreie Zeit der Patienten mit bronchopulmonalem Karzinoid deutet darauf hin, dass sich die bronchopulmonalen Karzinoide biologisch von den anderen 
NET unterscheiden. Darüber hinaus läuft in den USA eine offene Phase-II-Studie mit Everolimus plus Bevacizumab versus Standard-Chemotherapie (nach Wahl des Zentrums). Primärer Endpunkt ist die mediane Gesamtüberlebenszeit [26].

In der Planung befindet sich ein überregionales Register für das neuroendokrine Lungenkarzinom (PneuNET-Register). Ziel ist es, die aktuelle Datenlage bei den pulmonalen NET zu verbessern und dies für Diagnostik, Therapie und Forschung zu nutzen. Ein Schwerpunkt soll auf den Patienten mit fortgeschrittener Erkrankung liegen, wo die Datenlage besonders schlecht ist. Die Daten sollen prospektiv über eine gemeinsame Internet-Plattform mit dem bekannten NET-Register erfasst werden. Weitere Informationen zum PneuNETRegister können unter www.net-register.org bzw. direkt unter okan.zaba@elk-berlin.de abgerufen werden.

\section{Disclosure Statement}

Die Autoren waren Referenten beim «Dresdner Interdisziplinäres Gespräch 2013», das von Roche Pharma AG unterstützt wurde.

\section{Literatur}

1 Travis WD, Brambilla E, Müller-Hermelink HK, Harris CC (eds): World Health Organization Classification of Tumors. Pathology and Genetics of Tumours of the Lung, Pleura, Thymus and Heart, ed 1. Lyon, IARC Press, 2004.

2 Rindi G, Klöppel G, Alhman H, et al.: TNM staging of foregut (neuro)endocrine tumors: a consensus proposal including a grading system. Virchows Arch 2006;449:395-401.

- 3 Zahel T, Krysa S, Herpel E, et al.: Phenotyping of pulmonary carcinoids and a Ki-67-based grading approach. Virchows Arch 2012;460:299-308.

4 Schnabel PA: Großes Spektrum: Differentialdiagnostik neuroendokriner Tumore der Lunge ( $\mathrm{p}$ NET). Pneumol Nachr 2013;3:19-22.

$\checkmark 5$ Helmbold P, Lahtz C, Herpel E, et al.: Frequent hypermethylation of RASSF1A tumour suppressor gene promoter and presence of Merkel cell polyomavirus in small cell lung cancer. Eur J Cancer 2009;45:2207-2211.

6 Gottschling S, Jensen K, Herth FJF, Schnabel PA, Thomas M, Schnabel PA, Herpel E: Lack of prognostic significance of neuroendocrine differentiation and stem cell antigen co-expression in resected early-stage non-small cell lung cancer. Anticancer Res 2013;33:981-990.

7 Haug A, Auernhammer CJ, Wangler B, et al.: Intraindividual comparison of 68Ga-DOTA-TATE and 18F-DOPA PET in patients with NET. Eur $\mathrm{J}$ Nucl Mol Imaging 2009;36:765-770.

8 Esser JP, Krenning EP, Teunissen JJ, et al.: Comparison of [177Lu-DOTA(0), Tyr(3)]octreotate and [177Lu-DOTA(0),Tyr(3)]octreotide: which peptide is preferable for PRRT? Eur J Nucl Med Mol Imaging 2006;33:1346-1351.

9 Swärd C, Bernhardt P, Johanson V, et al.: Comparison of [177Lu-DOTA0,Tyr3]-octreotate and [177Lu-DOTA0,Tyr3]-octreotide for receptor-mediated radiation therapy of the xenografted human midgut carcinoid tumors GOT1. Cancer Biother Radiopharm 2008;23:114-120.
10 Kwekkeboom DJ, Bakker WH, Kooij PP, et al.: [177Lu-DOTAOTyr3]octreotate: comparison with [111In-DTPAo]octreotide in patients. Eur J Nucl Med 2001;28:1319-1325.

11 Bushnell DL, O'Dorisio TM, O'Dorisio S, et al.: 90Y-Edotreotide for metastatic carcinoid refractory to octreotide. J Clin Oncol 2010;28:1652-1659.

12 Cwikla JB, Sankowski A, Seklecka N, et al.: Efficacy of radionuclide treatment DOTATATE Y-90 in patients with progressive metastatic gastroenteropancreatic neuroendocrine carcinomas (GEP-NETs): a phase II study. Ann Oncol 2010;21:787-794.

13 Imhof A, Brunner P, Marincek N, et al.: Response, survival and long-term toxicity after therapy with the radiolabeled somatostatin analogue [90YDOTA]-TOC in metastasized neuroendocrine cancers. J Clin Oncol 2011;29:2416-2423.

14 Kwekkeboom DJ, de Herder WW, Kam BJ, et al.: Treatment with the radiolabeled somatostatin ana$\log$ [177Li-DOTA0,Tyr3]-octreotate: toxicity, efficacy and survival. J Clin Oncol 2008;26:2124-2130.

15 Teunissen JJ, Kwekkeboom DJ, Krenning EP: Quality of life in patients with gastroenteropancreatic tumors treated with [177Lu-DOTA0,Tyr3]octreotate. J Clin Oncol 2004;22:2724-2729.

16 Bodei L, Cremonesi M, Grana CM, et al.: Peptide receptor radionuclide therapy with $177 \mathrm{Lu}-\mathrm{DO}-$ TATATE: the IEO phase I-II study. Eur J Nucl Mol Imaging 2011;38:2125-2135

17 Valkema R, Pauwels SA, Kvols LK, et al.: Longterm follow-up of renal function after peptide receptor radiation therapy with [90Y-DOTA0,Tyr3]octreotide and [177Lu-DOTAo,Tyr3]-octreotate. J Nucl Med 2005;26:83S-91S

18 Claringbold PG, Brayshaw PA, et al.: Phase II of radiopeptide 177Lu-octreotate and capecitabin therapy of progressive disseminated neuroendocrine tumours. Eur J Nucl Mol Imaging 2011;38:302-311.
9 Claringbold PG, Price RA, Turner JH: Phase I-II study of radiopeptide $177 \mathrm{Lu}$-octreotate in combination with capecitabine and temozolomide in advanced low-grade neuroendocrine tumors. Cancer Biother Radiopharm 2012;27:561-569.

20 Kratochwil C, López-Benítez R, Mier W, et al.: Hepatic arterial infusion enhances DOTATOC radiopeptide therapy in patients with neuroendocrine liver metastases. Endocr Rel Cancer 2011;18: 595-602.

21 Arnold AM, Smylie M, Ding K, et al.: Randomized phase II study of vandetanib or placebo in smal cell lung cancer patients after complete or partial response to induction chemotherapy with or without radiation therapy: NCIC CTB BR.20. J Clin Oncol 2007;25:4278-4284.

22 Jotte R, Conkling P, Reynolds C, et al.: Randomized phase II trial of single-agent amrubicin or topotecan as second-line treatment in patients with small-cell lung cancer sensitive to first-line platinum-based chemotherapy. J Clin Oncol 2011;20: 287-293.

23 De Marinis F, Atmaca A, Tiseo M, et al.: Deacetylase inhibitor panobinostat in relapsed small cell lung cancer patients: Results of a multicenter phase II trial. Proc. ASCO 2010; e17521.

24 Le Treut J, Sault MC, Lena H, et al.: Multicentre phase II study of cisplatin-etoposide chemotherapy for advanced large-cell neuroendocrine lung carcinoma: the GFPC 0302 study. Ann Oncol 2013;24: 1548-1552.

25 clinicaltrials.gov/show/NCT00688623.

26 Dong M, Yao JC: mTOR inhibition, a potential novel approach for bronchial cacrinoids. Erc.endocrinology-journals.org/content/early/2011/03/22/ ERC-10-0290. full.pdf. 